\title{
Effect of deforolimus and VEGF on angiogenesis in endometrial stromal cells following three-dimensional culture
}

\author{
Jafar $\mathrm{Ai}^{1,2,3,4^{*}}$, Somayeh Ebrahimi ${ }^{5}$, Armin $\mathrm{Ai}^{6}{ }^{6}$ Roya Karimi ${ }^{1}$, Naghmeh Bahrami ${ }^{1}$ \\ ${ }^{1}$ Department of Tissue Engineering, Faculty of Advanced Technologies in Medicine, Tehran University of Medical Sciences, Tehran, \\ Iran; 'Corresponding Author: Jafar_ai@tums.ac.ir \\ ${ }^{2}$ Research Center for Science and Technology in Medicine, Tehran University of Medical Sciences, Tehran, Iran \\ ${ }^{3}$ Brain and Spinal Injury Research Center, Imam Hospital, Tehran University of Medical Sciences, Tehran, Iran \\ ${ }^{4}$ Cellular and Molecular Research Center, Tehran University of Medical Sciences, Tehran, Iran \\ ${ }^{5}$ Department of Biological Science, Faculty of Biology, Kharazmi University (TMU), Tehran, Iran \\ ${ }^{6}$ Faculty of Dentistry, Tehran University of Medical Sciences, Tehran, Iran
}

Received 4 November 2012; revised 3 December 2012; accepted 2 January 2013

\begin{abstract}
The presence of endometrial tissue outside of the uterine cavity is named endometriosis and is the most common gynecologic disorder in women. Determining the inhibitory effect of a Deforolimus on angiogenesis in a three-dimensional (3-D) culture of human endometrial stromal cells (hEnCs) in vitro. The important mechanism in the pathogenesis of endometriosis is angiogenesis, and deforolimus has been shown to have anti-angiogenic activity. This was an in vitro study of human endometrial stromal cells in 3-D culture of fibrin matrix. Endometrial stromal cells isolated and placed in a 3-D fibrin matrix culture system for angiogenesis with VEGF and inhibit angiogenesis by deforolimus. Finally these cells analyzed by CD31 antibodies. After 3 weeks, in cells treated with VEGF, endothelial cell branching was observed and rudimentary capillary-like structures formed. In the presence of $5 \mu \mathrm{M}$ of deforolimus, angiogenesis was reduced. The deforolimus were shown to be effective in inhibiting the mechanisms of angiogenesis.
\end{abstract}

Keywords: Endometrial Stromal Cells;

Endometrium; 3-D culture; Deforolimus; VEGF

\section{INTRODUCTION}

Endometriosis is a chronic benign gynecological disorder characterized by presence of endometrial cells throughout the uterus or peripheral organs and tissues. The rate of occurrence of endometriosis is about $11 \%$ in all women and is usually identified in women with pelvic pain and infertility. The etiology and pathogenesis of endometriosis is not clearly understood, but there are a number of theories. Historically theories have proposed different reasons for endometriosis including retrograde menstruation, coelomic metaplasia, embryonic rest and lymphovascular metastasis. However, several predisposing factors ranging from genetic to environmental factors as well as physiological and anatomical defects have been reported in correlation with it $[1,2]$. The human endometrium develops new capillaries from existing microvessels, i.e. angiogenesis, which then undergoes maturation and remodeling [3]. Excessive endometrial angiogenesis is suggested to be an important mechanism in the pathogenesis of endometriosis [4]. Recent progresses in the field of stem cells and evidences for in situ presence of endometrial stem cells or migratory stem cells from bone marrow have opened up a new way toward stem cell-based pathogenesis of endometriosis. Stem cells (SCs) are undifferentiated cells, which are can remain at this state for several generations following cell proliferations. SCs are also able to take part in natural in vivo phenomena such as wound repair or tissue regenerations after physical damages. Today, stem cells are being considered as an important tool in cell therapy which is a novel therapeutic method by clinical researchers [5,6]. On this basis, endometrial stem cells (EnSCs) with higher ability for proliferation, differentiation and fast angiogenesis during menstruation alongside with their immune tolerance for embryo during pregnancy have been considered as a valuable source of stem cell $[7,8]$. Additionally our previous studies demonstrated that EnSCs are exceptionally capable of participating in a phenomenon like angiogenesis in 3-D cultures which is similar to early stages of 
endometriosis [9-11] and may propose a novel mechanism in pathogenesis of endometriosis compared to the traditional theories that emphasize on retrograde menstruation as a possible factor. These studies are supported by others who demonstrated recruitment of bone marrow-derived mesenchymal stem cells to the endometrium [12] and these all may point to the role of stem cells in pathogenesis of endometriosis $[13,14]$. Therefore, endometrial stem cells could be considered as binary role-players in helping endometrium function naturally and also in endometriosis. They potentially could be used as targets in endometriosis therapy. For this, rapamycin was extracted from a bacterium, Streptomyces hygroscopicus, taken from the soil of Rapa Nui. Thereafter, there are three rapamycin analogs that are used as anti-tumor agents: CCI779 (Temsirolimus), AP23573 and RAD001 (Everolimus) [15]. Rapamycin and its analogs bind to the cellular protein FK506 through binding protein 12 (FKBP12), and directly inhibit mTOR (mammalian Target of Rapamycin), a cellular enzyme that plays a key role in cell growth and proliferation [16,17]. Rapamycin appears to inhibit angiogenesis [18], which is consistent with the observation that PI3K signaling is required for expression and secretion of the pro-angiogenic vascular endothelial growth factor (VEGF) from endothelial cells [16]. Deforolimus has shown promise in treating a broad range of tumors, especially sarcomas. Deforolimus has been shown to inhibit the secretion of VEGF in cell lines, suggesting that it exerts some of its anti-tumor effects through inhibition of angiogenesis [19].

In our previous studies we showed that in vitro culture of human endometrial tissue in a 3-D fibrin matrix could proliferate and sprout new vessels [9-11]. In this study EnSCs were differentiated to endothelial cells $\left(\mathrm{CD} 31^{+}\right)$. The aim of the present study was to determine the potential inhibitory effect of a deforolimus (AP23573; Sig$\mathrm{ma}$ ) on angiogenesis in an in vitro three-dimensional fibrin matrix culture of human endometrial stromal cells treated with VEGF $\left(\mathrm{CD} 31^{+}\right)$.

\section{MATERIALS AND METHODS}

Endometrial biopsies were obtained from cases referred to the hospital for infertility treatments. A written informative consent form describing the procedures and aims of the study was taken from each donor in compliance with regulations concerning the use of human tissues. The biopsied tissues were washed in Dulbecco's phosphate buffered saline (DPBS), minced and treated with collagenase I type A $(2 \mathrm{mg} / \mathrm{ml})$ for $30-45 \mathrm{~min}$ at $37^{\circ} \mathrm{C}$ with agitation. Following tissue digestion, epithelial and stromal cells were separated using filtration. The cells were then centrifuged and underwent Ficoll purification. The isolated cells were cultured in DMEM/F12 medium containing $10 \%$ FBS, $1 \%$ antibiotic penicillin/streptomycin and $1 \%$ Glutamine and then incubated at $37^{\circ} \mathrm{C}$ in $5 \% \mathrm{CO}_{2}[20]$.

\subsection{Phenotypic Characterization}

Having three passages after isolation, the cells were characterized by flow cytometry for cell surface markers. In order to do so, cells were washed with HBSS $+2 \%$ BSA two times and incubated with the specific antibody at the concentrations recommended by the manufacturers. Cells were incubated for 20 minutes and analyzed by flow cytometry. Following antibodies were used: FITCconjugated anti-CD90, FITC-CD146, PE anti-human CD 105 (mesenchymal markers), FITC-conjugated anti-CD34 (hematopoietic marker), PE anti-human CD31 (endothelial marker), FITC-conjugated mouse IgG1, and PE-conjugated mouse IgG1 were used for negative control, all from Santa Cruz. For intracellular staining by the antibody, cells were washed twice in PBS with 2\% BSA and fixed with $4 \%$ paraformaldehyde (PFA) for $30 \mathrm{~min}$. Subsequently cells were washed twice in $0.5 \%$ Tween 20 and $0.1 \%$ Triton X-100 in PBS (T-PBS). Primary antibodies were added and incubation was performed for $30 \mathrm{~min}$. Cells were then washed twice in PBS. Secondary antibodies were subsequently diluted in PBS at the concentrations suggested by the manufacture instructions. Cells were analyzed using flow cytometry and OCT4 was used as the intracellular antibody (Abcam, USA).

\subsection{Three-Dimensional in Vitro Culture of Endometrial Fragments}

sEnSCs were examined to be free from endothelial cells using CD31 staining. The cells were then mixed with $1 \mathrm{ml} /$ well fibrinogen solution (3 $\mathrm{mg} / \mathrm{ml}$ in M199 culture medium), plated in culture dishes (24 wells) and 15 ul thrombin (50NIH unit/ml in $0.15 \mathrm{M} \mathrm{NaCl}$ ) was added. After gel formation, each well was covered with 1 $\mathrm{ml}$ M199 supplemented with $5 \%$ fetal bovine serum (FBS), $0.1 \%$ e-amino-glutamine and $100 \times$ antibiotic solutions and VEGF $50 \mathrm{ng} / \mathrm{ml}$. The cells were cultured at $37^{\circ} \mathrm{C}$ in $5.5 \% \mathrm{CO}_{2}$ and $95 \%$ humidity for time period up to 3 weeks. The medium was changed every 3 days and cultures were observed twice weekly with an inverted microscope for visual evidences of angiogenesis. After 3 weeks, we used EnSCs that were treated with VEGF before and the expression of CD31 was confirmed in these cells prior to being treated with $5 \mu \mathrm{M}$ of deforolimus for 1 week in 3-D culture.

\subsection{Morphological Observation}

Cells were observed under a phase-contrast microscope in order to have their overall appearance be eva- 
luated. Microphotographs were taken with $10 \times$ objective (TS-100 Nikon, Japan).

\subsection{Immunocytochemistry Analysis}

Day 21 post treatment (PT) cells for CD31 antibody as an endothelial cells marker were fixed with 4\% PFA (Sigma-Aldrich) for $30 \mathrm{~min}$ at room temperature. After permeabilization with $0.2 \%$ Triton-X 100 (Sigma-Aldrich) for 10 min the cells were blocked with goat serum and incubated overnight with primary antibody CD31, mouse monoclonal anti-human, Abcam, 1:200 at $4^{\circ} \mathrm{C}$ and 2 h with secondary antibody (Alexa Fluor@488 donkey anti mouse IgG, at a 1:500 dilution; Abcam, USA) at $37^{\circ} \mathrm{C}$. For negative controls, only the secondary antibody was used. Slides were washed with PBS between each step and nuclei staining were performed using 4', 6-diamidino-2-phenylindole (DAPI, Sigma). Cells were examined by fluorescence microscope (Olympus BX51, Japan).

\subsection{Flow Cytometry Analysis}

After 3 weeks, EnSCs treated with VEGF were prepared for flow cytometry for CD31 (endothelial cells marker). Then VEGF treated EnSCs were treated with deforolimus after 3 weeks and were tested for CD31 byflow cytometry. Cells were trypsinized and washed with staining buffer, which contained PBS with 3\% FBS and 0.05\% sodium azide. Cell pellets were incubated on ice with CD31 conjugated directly with PE (Abcam) for 1 hour. Cells were washed 3 times by centrifugation at $400 \mathrm{~g}$ for 5 minutes and were resuspended in $500 \mu \mathrm{l}$ to $1 \mathrm{ml}$ of ice cold PBS, $10 \%$ FCS, $1 \%$ sodium azide. Cells were kept in the dark on ice or in a fridge at $4^{\circ} \mathrm{C}$ until analysis.

\section{RESULTS}

\subsection{Characterization of Isolated Human EnSCs}

The immunophenotype was based on the flow cytometry analysis of a subset of embryonic stem cell marker (OCT4), mesenchymal stem cell markers (CD90, CD146 and CD105), hematopoietic marker (CD34) and endothelial marker (CD31). The flow cytometric analysis showed that the EnSCs were positive for CD90, CD105, OCT4 and were negative for CD31, CD34 (Figure 1).
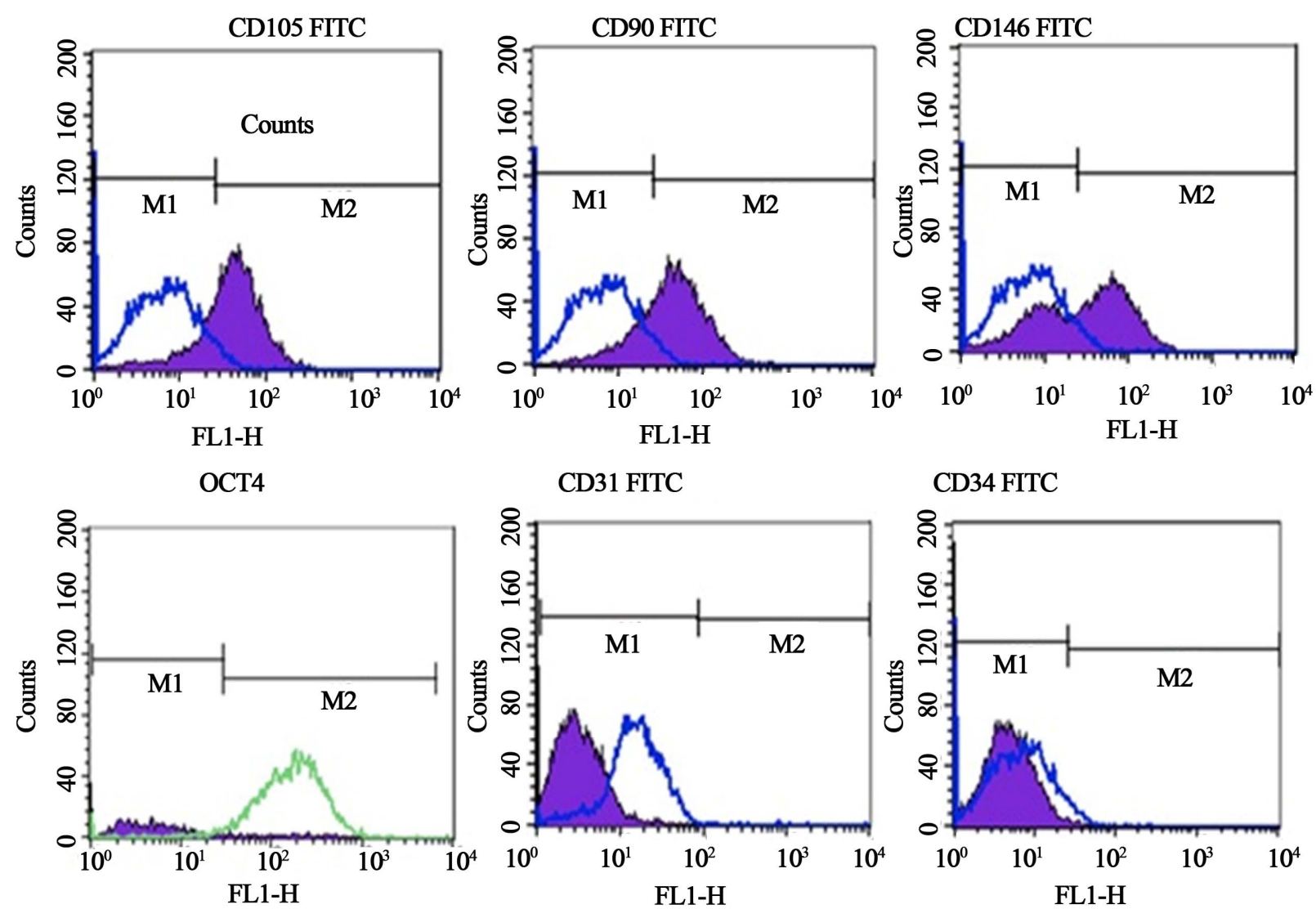

Figure 1. Flow cytometric analysis of isolated EnSCs for mesenchymal stem cell markers (CD90, CD146 and CD105), hematopoietic marker (CD34), endothelial marker (CD31) and embryonic stem cell marker (OCT4). As shown in figure 2 the isolated cells are positive for CD90, CD146, CD105, and OCT4 and are negative for CD31, CD34. 


\subsection{Morphological Differentiation, Immunocytochemistry and Flow Cytometry Analysis}

The main cellular event observed during the first week of culture of EnSCs with VEGF was formation of sheets of cells in the fibrin matrix (Figure 2(A)). From the beginning of the third week, angiogenesis was observed as tube-like structures sprouting into the 3-D fibrin matrix and were observed in the outgrowths and grew independently until the end of culture period (Figure 2(A)). Immunocytochemistry analysis showed that endometrial stromal cells after treated with VEGF for 3weeks express endothelial marker CD31 (Figure 3). Flow cytometry analysis of these structures was $15.57 \%$ positive for CD31 as a marker of endothelial cells (Figure 4(a)). During the 1st week of culture, invasion of deforolimus treated CD31 positive EnSCs occurred into the fibrin matrix in the control group and outgrowths were reduced to $11.64 \%$. The effect of deforolimus was witnessed on angiogenesis in the experimental groups. In presence of 5 $\mu \mathrm{M}$ deforolimus, angiogenesis was markedly reduced compared to the control wells (Figure 2(C)). The results of flow cytometry for CD31, confirmed morphological results (11.64\%, Figure 4(b)).
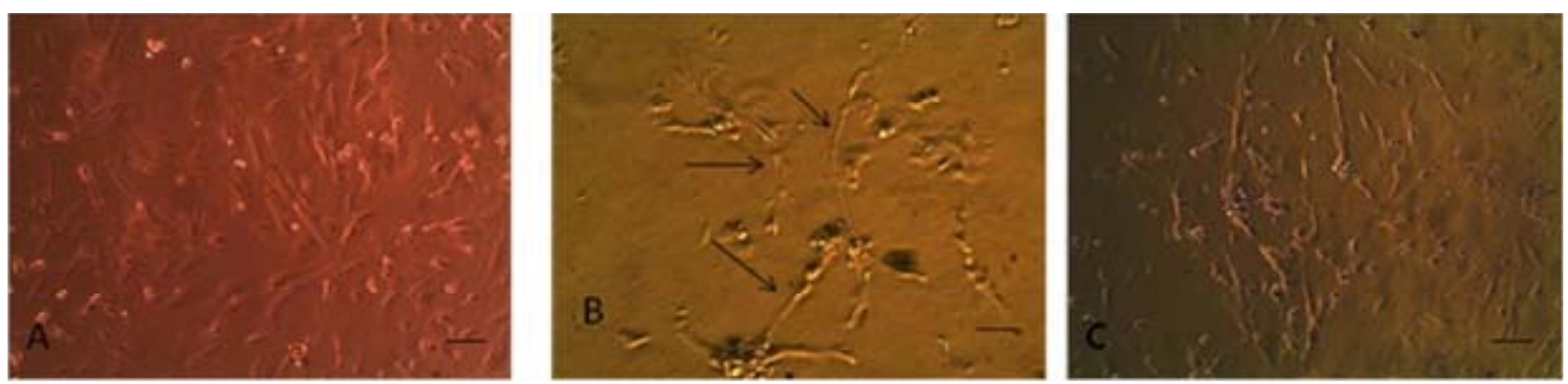

Figure 2. Microscopic phase-contrast photograph of endometrial stem cells formed sheets of cells in the fibrin matrix after 1 week (A); Angiogenesis in the three-dimensional fibrin matrix after 3 weeks. Tube-like structures sprouting into the fibrin matrix (B); Inhibition of angiogenesis in the presence of $5 \mu \mathrm{M}$ of deforolimus (C). Scale bar: $100 \mu \mathrm{m}$.
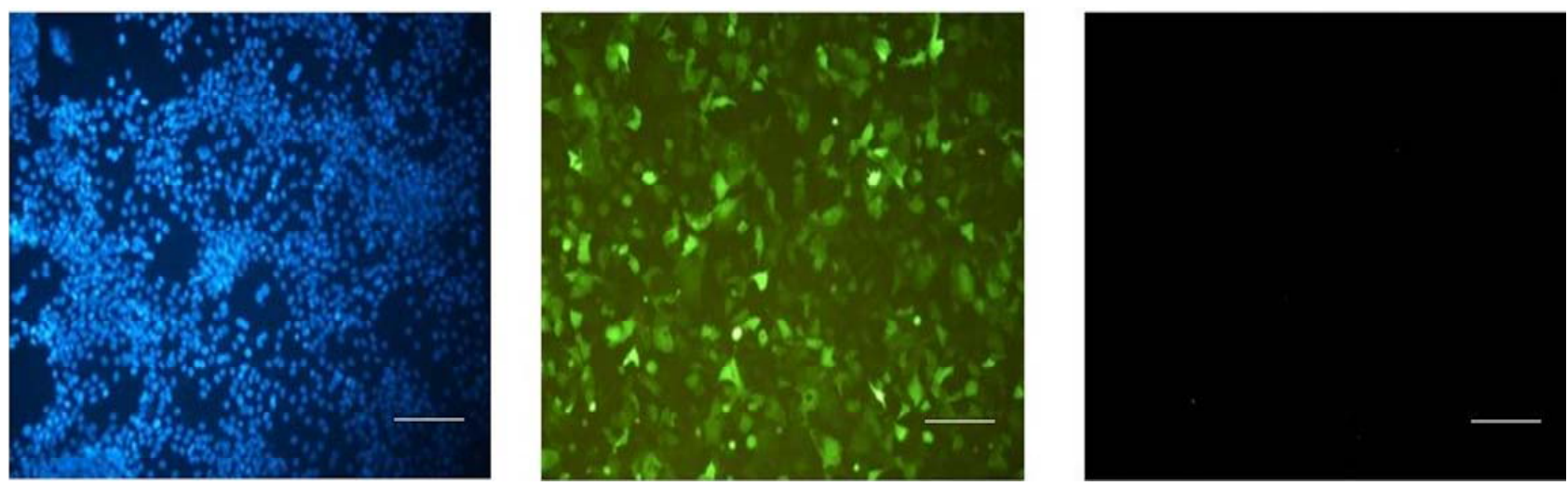

Figure 3. immunocytochemistry analysis for endothelial marker CD31 after exposure endometrial stem cell with VEGF. Scale bar: $100 \mu \mathrm{m}$.

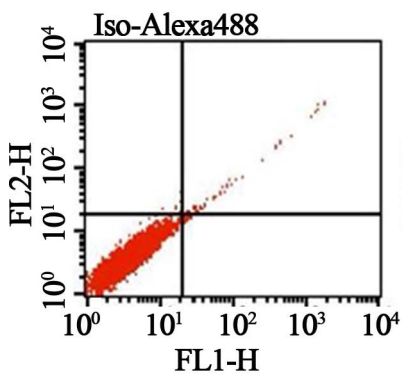

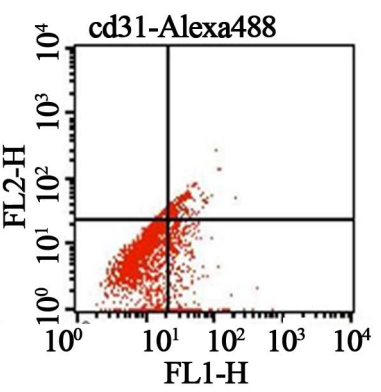

(a)

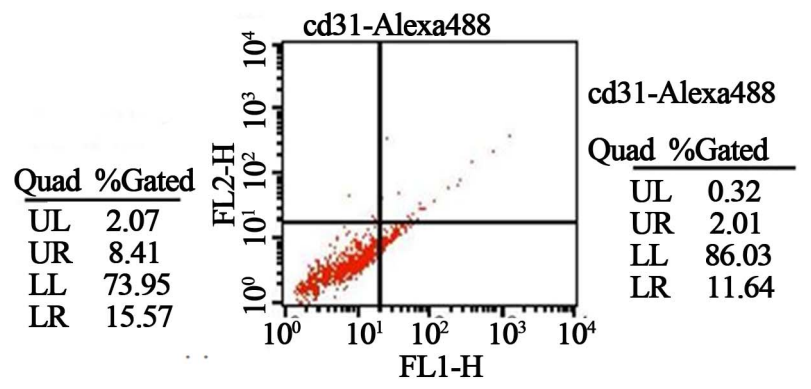

(b)

Figure 4. Flow cytometric analysis for CD31 in treatment group with VEGF (a) and treatment group with deforolimus (b). 


\section{DISCUSSION}

In the present study, in the first step, we have utilized 3 -D in vitro fibrin matrix for culturing isolated endometrial stromal cells in presence of VEGF for angiogenesis. Using this model, we have demonstrated threedimensional cell proliferation and invasion, resulted in generation of new vessels. These cells were $15.57 \%$ positive for CD31. In the second step, we treated these cells $\left(\mathrm{CD} 31^{+}\right)$with deforolimus at concentration of $5 \mu \mathrm{M}$ in 3D culture. The results show that deforolimus reduced tube-like structures and caused $11.64 \%$ decrease in CD31 expression. We found that deforolimus inhibited angiogenesis in these cells and can be used for endometriosis patients. The pathogenesis of endometriosis remains poorly understood, mostly because the initial stages of the disease are neither detectable nor observable in humans. By the time that endometriosis is brought to medical attention, the disease already is well established. In addition, this disease occurs only in humans and subhuman primates, since menstrual shedding is a requirement for its spontaneous development $[1,2]$. Endometrial angiogenesis suggests the possibility for novel medical treatments aimed at the inhibition of angiogenesis, which is similar to recently discussed concepts for cancer treatment [21, 22]. Our findings suggest that deforolimus, an mTOR inhibitor, can negatively modulate both cell proliferation and angiogenesis in a concentration dependent manner. Deforolimus is a potent inhibitor of VEGF expression, which is most probably the mechanism behind the diminished blood-vessel formation. mTOR is a serine/threonine kinase that is a central regulator of cell growth, proliferation and metabolism in response to environmental and nutritional cues. It is a critical effector of signals mediated by the phosphatidylinositol 3-kinase (PI3K) pathway, a signaling cascade that is important in tumors [16]. Activation of PI3K by growth factors signals the cells to grow and proliferate; this signal is transmitted through the cell by AKT to mTOR. Deforolimus is known to cross the blood-brain barrier (BBB) in experimental animals, suggesting that it may be useful for treating brain cancer [23]. In addition to its cytostatic activity, Deforolimus has been shown to reduce tumor size in mouse models and to reduce cell size in human tumor cell lines $[19,23,24]$. To define its potential for being used in combination chemotherapy regimens, Deforolimus was investigated along with several chemotherapeutic agents in cell proliferation assays [25]. Deforolimus has been shown to inhibit the secretion of VEGF in cells, suggesting its anti-tumor effects through inhibition of angiogenesis [19]. Together, these preclinical results served as the basis for testing deforolimus in Phase 1 clinical trials in advanced cancer patients. Further in vitro and in vivo studies are required to assess the potential of different anti-angiogenic drugs to inhibit the initiation or progression of endometriosis.

\section{CONCLUSION}

In our previous studies we found, endometrial stem cells can differentiate to other cells such as endothelial cells, neuron, adipocyte, osteoblast and myoblast [26-30] and based on our findings, in this research, it seems that sloughed stromal cells as basic factor in angiogenesis causing pelvic fibrin adhesions in a retrograde manner would be able to proliferate as the first step of endometriosis. Furthermore, studying in vitro (3-D) models shows stromal cells proliferation and angiogenesis. These two phenomena are considered crucial for the development of the angiogenesis in endometriotic lesions and deforolimus were shown to be effective in inhibiting the mechanisms of angiogenesis.

\section{ACKNOWLEDGEMENTS}

We thank Tehran University of Medical Sciences Research assistant, Iranian Council of Stem Cell Technology and Iran National Science Foundation (INSF).

\section{REFERENCES}

[1] Kettel, L.M. and Hummel, W.P. (1997) Modern medical management of endometriosis. Obstetrics and Gynecology Clinics of North America, 24, 361-367. doi:10.1016/S0889-8545(05)70309-0

[2] Adamson, G.D. and Nelson, H.P. (1997) Surgical treatment of endometriosis. Obstetrics and Gynecology Clinics of North America, 24, 375-409. doi:10.1016/S0889-8545(05)70310-7

[3] Norrby, K. (1997) Angiogenesis-New aspects relating to its initiation and control. Acta Pathologica, Microbiologica et Immunologica Scandinavica, 105, 417-437. doi:10.1111/j.1699-0463.1997.tb00590.x

[4] Healy, D.L., Rogers, P.A.W., Hii, L. and Wingfield, M. (1998) Angiongenesis: A new theory for endometriosis. Human Reproductive Update, 14, 736-740. doi:10.1093/humupd/4.5.736

[5] Kim, S.U. and Vellis, J. (2009) Stem cell based cell therapy in neurological diseases: A review. Journal of Neuroscience Research, 87, 2183-2200. doi:10.1002/jnr.22054

[6] Pucéat, M. (2006) Stem cell therapy in heart failure: Where do we stand and where are we heading? Heart Failure Monitor, 5, 44-49.

[7] Gargett, C.E., Schwab, K.E., Zillwood, R.M., Nguyen, H.P.T. and Wu, D. (2009) Isolation and culture of epithelial progenitors and mesenchymal stem cells from human endometrium. Biology Reproduction, 80, 1136-1145. doi:10.1095/biolreprod.108.075226

[8] Meng, X., Ichim, T. and Zhong, J. (2007) Endometrial regenerative cells: A novel stem cell population. Journal of Translational Medicine, 5, 57-63. 


\section{doi:10.1186/1479-5876-5-57}

[9] Esfandiari, N., Ai, J., Bielecki, R., Gotlieb, L. and Casper, R.F. (2007) Expression of glycodelin and cyclooxygenase-2 in human endometrial tissue following three-dimensional culture. American Journal of Reproductive Immunology, 57, 49-54.

doi:10.1111/j.1600-0897.2006.00445.x

[10] Esfandiari, N., Khazaei, M., Ai, J., Bielecki, R., Gotlieb, L. and Ryan, E. (2007) Effect of a statin on an in vitro model of endometriosis. Fertility and Sterility, 87, 257262. doi:10.1016/j.fertnstert.2006.06.040

[11] Esfandiari, N., Khazaei, M., Ai, J., Nazemian, Z., Jolly, A. and Casper, R. (2008) Angiogenesis following three-dimensional culture of isolated human endometrial stromal cells. International Journal of Fertility Sterility, 2, 19-22.

[12] Taylor, R.N., Lundeen, S.G. and Giudice, L.C. (2002) Emerging role of genomics in endometriosis research. Fertility and Sterility, 78, 694-698. doi:10.1016/S0015-0282(02)03325-3

[13] Figueira, P.G., Abrão, M., Krikun, G. and Taylor, H.S. (2011) Stem cells in endometrium and their role in the pathogenesis of endometriosis. Annals of the New York Academy of Sciences, 1221, 10-17. doi:10.1111/j.1749-6632.2011.05969.x

[14] Sasson, I.E. and Taylor, H.S. (2008) Stem cells and the pathogenesis of endometriosis. Annals of the New York Academy of Sciences, 1, 106-115. doi:10.1196/annals.1434.014

[15] McGurl, D. (2005) From easter island soil to treating sarcoma rapamycin derivatives. ESUN, 2, 14-19.

[16] Schieke, S.M., Phillips, D., McCoy, J.P., Aponte, A.M., Shen, R.F., Balaban, R.S. and Finkel, T. (2006) The mammalian target of rapamycin (mTOR) pathway regulates mitochondrial oxygen consumption and oxidative capacity. Journal of Biological Chemistry, 281, 27643-27652. doi:10.1074/jbc.M603536200

[17] Vignot, S., Faivre, S., Aguirre, D. and Raymond, E. (2005) mTOR-targeted therapy of cancer with rapamycin derivatives. Annals Oncology, 16, 525-537. doi:10.1093/annonc/mdi113

[18] Guba, M., Breitenbuch, P., Steinbauer, M., Koehl, G., Flegel, S. and Hornung, M. (2002) Rapamycin inhibits primary and metastatic tumor growth by antiangiogenesis: Involvement of vascular endothelial growth factor. $\mathrm{Na}$ tional Medicine, 8, 128-135. doi:10.1038/nm0202-128

[19] DuBois, S., Marina, N. and Glade-Bender, J. (2010) Angiogenesis and vascular targeting in ewing sarcoma: A review of preclinical and clinical data. Cancer, 116, 749757. doi:10.1002/cncr.24844

[20] Mobarakeh, T.Z., Ai, J., Yazdani, F., Rezayat, M., Ghanbari, Z., NorooziJavidan A., Massumi, M. and Ebrahmi, S. (2012) Human endometrial stem cells as a new source for programming to neural cells. Cell Biology International Report, 19, 7-14. doi:10.1042/CBR20110009

[21] Folkman, J. (2006) Antiangiogenesis in cancer therapy endostatin and its mechanisms of action. Experimental Cell Research, 312, 594-601. doi:10.1016/j.yexcr.2005.11.015

[22] Ai, J. and Mehrabani, D. (2010) Are endometrial stem cells novel tools against ischemic heart failure in women? A hypothesis. Iran Red Crescent Medicine Journal, 12, 73-75.

[23] Metcalf, C.A., Bohacek, R., Rozamus, L.W., Burns, K.D., Roses, J.B. and Rivera, V.M. (2004) Structure-based design of AP23573, a phosphorous-containing analog of rapamycin for anti-tumor therapy. Cancer Research, 45, 2476-2480

[24] Clackson, T., Metcalf, C.A., Rivera, V.M., Knowles, H.L., Tang, H. and Burns, K.D. (2003) Broad anti-tumor activity of ap23573, an mTOR inhibitor in clinical development. American Society of Clinical Oncology, 22, 882.

[25] Rivera, V.M., Kreisberg, J.I., Mita, M.M., Goldston, M., Knowles, H.L. and Herson, J. (2005) Pharmacodynamic study of skin biopsy specimens in patients (patients) with refractory or advanced malignancies following administration of AP23573, an mTOR inhibitor. American Society of Clinical Oncology, 23, 3033.

[26] Ai, J., Esfandiari, N. and Casper, R. (2009) Detection of aromatase in human endometrial tissue cultured in threedimensional fibrin matrix in vitro. Iranian Journal of Reproductive Medicine, 7, 105-109.

[27] Ai, J., Noroozi, A. and Mehrabani, D. (2010) The possibility of differentiation of human endometrial stem cells into neural cells. Iranian Journal of Reproductive Medicine, 12, 328-331.

[28] Ai, J., et al. (2012) Derivation of adipocytes from human endometrial stem cells (EnSCs). Journal of Reproduction and Infertility, 13, 151-157.

[29] Ai, J. and Mehrabani, D. (2010) The potential of human endometrial stem cells for osteoblast differentiation. Iranian Journal of Reproductive Medicine, 12, 585-587.

[30] Ai, J., Tabatabaei, F.S. and Kajbafzadeh, A.M. (2009) Myogenic potential of human endometrial adult stem cells. Iranian Journal of Medical Hypotheses and Ideas, 3, 3-6. 